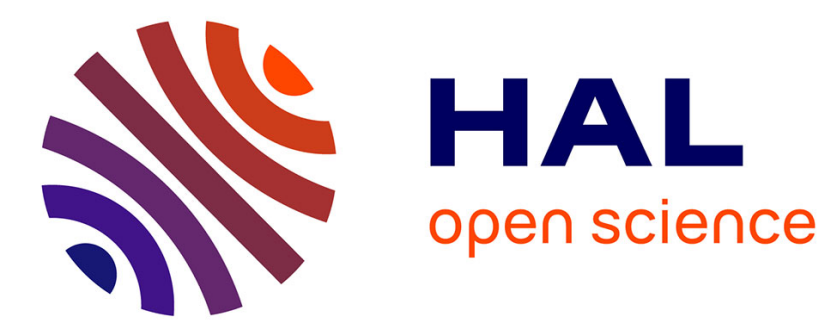

\title{
Comme un rayon de lumière à travers les ténèbres
}

\author{
Michel Bourdeau
}

\section{To cite this version:}

Michel Bourdeau. Comme un rayon de lumière à travers les ténèbres: La correspondance de Mill et de Gustave D'Eichthal (1829-1871). Cahiers philosophiques, 2017, 148, pp.105-108. halshs-01411168

\section{HAL Id: halshs-01411168 \\ https://shs.hal.science/halshs-01411168}

Submitted on 13 Dec 2016

HAL is a multi-disciplinary open access archive for the deposit and dissemination of scientific research documents, whether they are published or not. The documents may come from teaching and research institutions in France or abroad, or from public or private research centers.
L'archive ouverte pluridisciplinaire HAL, est destinée au dépôt et à la diffusion de documents scientifiques de niveau recherche, publiés ou non, émanant des établissements d'enseignement et de recherche français ou étrangers, des laboratoires publics ou privés. 


\title{
Comme un rayon de lumière à travers les ténèbres ${ }^{1}$ \\ LA CORRESPONDANCE DE MILL ET DE GUSTAVE D’EICHTHAL (1829-1871)
}

\author{
Michel Bourdeau IHPST (CNRS-Paris1-ENS)
}

\begin{abstract}
Mes relations avec Mill (...) ont eu une grande influence sur sa vie (luimême l'atteste) et aussi sur la mienne. Dans une certaine mesure, c'est lui qui m'a ouvert l'Angleterre comme je lui ai ouvert la France. Ce qui nous rapprochait, ce n'étaient point des idées abstraites. C'était notre nature et nos désirs d'apôtres.

Gustave d'Eichthal au Dr. Henry, 26 nov 73.
\end{abstract}

\section{PRÉSENTATION}

Gustave d'Eichthal (1804-1886), ainsi que son frère cadet Adolphe (1806-1875), comptent parmi les grandes figures du saint-simonisme ${ }^{2}$. Leur père, Louis d'Eichthal, est un banquier juif venu d'Allemagne s'établir à Paris en 1812, après quelques années passées à Nancy, et qui se convertit au catholicisme en 1817. Alors qu'Adolphe s'illustre à son tour dans la banque, aux côtés des frères Pereire, Gustave, en qui son père aurait aimé voir son successeur, choisit une autre voie et déploie son activité sur une multitude de fronts, qui se laissent rattacher, à un degré ou à un autre, à son appartenance à la «famille saintsimonienne ». Il est secrétaire de la Société ethnologique de Paris, fondée en 1839 par W.F. Edwards et publie dans le bulletin de cette société une longue étude sur les Foulhas, ou Fellans, dont il fait un livre en 1841. Le rapport avec le saint-simonisme passe alors par le mulâtre Ismail Urbain, les Lettres sur la race blanche et la race noire (1839) reprenant la correspondance échangée entre les deux amis.

C'est aussi un grand philhellène. Othon, le premier souverain de la Grèce devenue indépendante, est un membre de la famille royale de Bavière, dont l'oncle de Gustave, Simon d'Eichthal, se trouve être le banquier. C'est ainsi que, de 1833 à 1835, le jeune homme est en Grèce pour participer à la mise en place des finances du royaume. Le rapport au saint-simonisme est là encore immédiat, et passe cette fois par ce que Michel Chevalier appelait le Système de la Méditerranée. Quelque trente ans plus tard, quand il participe à la fondation de «l'Association pour l'encouragement des études grecques en France », c'est dans l'idée de faire du grec la langue internationale de l'Europe.

Si donc, pour d'Eichthal, le saint-simonisme est le centre d'où tout rayonne, cela ne signifie pas que son adhésion ait été immédiate. Jusqu'en 1828, c'est d'abord un disciple, et même le premier disciple, d'Auguste Comte qui, en 1822, avait été chargé de compléter la formation mathématique des deux frères. C'est même Comte qui introduit Gustave à SaintSimon, en 1823 ; mais à l'époque, semble-t-il, le courant ne passe pas : " Je l'évitais, le regardant comme une espèce de fou, avec lequel on ne pouvait se trouver en contact sans se compromettre »(Le Bret 120 ; cf. Ratcliffe 133). Cinq ans plus tard, en Angleterre, c'est

\footnotetext{
${ }^{1}$ Lettre du 30 novembre 1831, infra p. **.

2 Cette présentation emprunte la plupart des données biographiques concernant Gustave d'Eichthal à :

- Hervé Le Bret : Les frères d'Eichthal, presses de l'université Paris-Sorbonne, 2012, (abrégé en : Lebret)

- Barrie M Ratcliffe et W H Chaloner : A French sociologist looks at Britain, Gustave d'Eichthal and British

Society in 1828, Mauchester UP, 1977 (abrégé en :Ratcliffe).
} 
comme disciple d'Auguste Comte qu'il se présente en Grande-Bretagne ${ }^{3}$; et c'est encore à Comte qu'il rend compte de son voyage (Ratcliffe 7-9).

Le séjour des deux frères en Angleterre les éloigne toutefois de Comte. A leur retour, Olindes Rodrigues, le dernier secrétaire de Saint-Simon, qui est aussi un proche de la famille d'Eichthal (Gustave épousera Cécile Rodrigues, lointaine parente d'Olindes), présente Gustave à Enfantin, qui travaille alors à la Caisse hypothécaire. C'est le déclic, qui coïncide avec le moment où le saint-simonisme prend son essor ${ }^{4}$. Gustave devient un prosélyte infatigable, jusqu'à la dispersion, en 1833. Avec Isaac Pereire, il s'occupe des finances du mouvement, et même le finance en partie, avant de s'en éloigner en novembre 1832, sur les demandes instantes de sa famille (Ratcliffe140). Dans les conflits qui divisèrent le mouvement, il se range aux côtés d'Enfantin, dont il restera toujours très proche. A la mort de ce dernier, en 1864, il est un de ses six légataires ; mais, vexé de n'y figurer qu'en troisième place, il décide de constituer son propre fonds d'archives. Déposé par la suite à la Bibliothèque de l'Arsenal et à la Bibliothèque Thiers, ce fonds constitue une des sources les plus importantes pour l'histoire du saint-simonisme. A sa mort, en 1886, il laissera inachevée une biographie de Saint-Simon, qu'il avait commencée deux ans plus tôt.

D'Eichthal et Mill ont fait connaissance à Londres en 1828. Louis d'Eichthal y avait envoyé ses deux fils parfaire leur formation ${ }^{5}$. Ce voyage, qui dura plus de six mois (avrilnovembre 1828) a joué un rôle déterminant dans l'évolution ultérieure du jeune homme. Non seulement il contribua à l'éloigner de Comte, mais il lui permit d'acquérir des connaissances utiles pour son essai sur les finances publiques (1829) qui attireront sur lui l'attention des saint-simoniens. Il comptait également en tirer un ouvrage sur l'Angleterre. Si le projet ne se réalisera pas, malgré les encouragements de Mill, il en rapporta des notes et un journal publiés en 1902 par son fils Guillaume et qui contiennent une analyse remarquable de la société anglaise alors en cours d'industrialisation (Ratcliffe, 13-109).

C'est aussi lors de ce séjour que se noua l'amitié avec Stuart Mill, amitié qui devait compter pour l'un comme pour l'autre. A cette date, Mill avait déjà vécu en France. A l'âge de quatorze ans, il avait été envoyé pendant un an par son père à Montpellier, chez un frère de Bentham qui y était négociant, et il a toujours gardé de ce séjour un souvenir très vivace. A Paris, il avait aussi fréquenté le salon de Jean-Baptiste Say et y avait même croisé SaintSimon. Il était donc comme prédisposé à sympathiser avec le jeune voyageur venu de Paris. La correspondance qui s'ensuivit, et qui s'étend sur plus de quarante ans (de 1829 à 1871), est riche d'un double enseignement. Tout d'abord, elle nous rappelle la place éminente qu'occupait la France dans la pensée, et le cœur, du philosophe anglais (Mill est enterré en Avignon). En nous montrant le puissant attrait exercé un temps par le saint-simonisme sur son esprit, elle nous oblige à renoncer à une interprétation simpliste de son libéralisme et nous aide à mieux apprécier la richesse de sa pensée socio-politique.

Dans le premier cas, il est bien connu que les amitiés françaises ont beaucoup compté pour Mill. Outre d'Eichthal, il a correspondu avec les esprits les plus illustres d'alors : Comte,

\footnotetext{
3 En 1824, quand il réédite, sous le titre de Système de politique positive, le Plan des travaux scientifiques nécessaires à la réorganisation de la société (1822), Comte se présente encore, pour des raisons diplomatiques, comme le disciple de celui avec lequel il vient pourtant de rompre. Deux ans après, il publie encore dans des revues saint-simoniennes. Aux yeux du public, il appartient à la nébuleuse saint-simonienne, comme le prouve la quinzième séance de l'Exposition de la doctrine de Saint-Simon (première année, 18281829).

4 La correspondance qu'il échange avec Comte en décembre 1829 montre assez bien la nature affective des motifs qui l'ont poussé vers le saint-simonisme : il se déclare insatisfait par le caractère exclusivement intellectuel des positions comtiennes. Voir encore sa déposition lors du procès des saint-simoniens, Ratcliffe p. 137-38, n. 117.

5 . Dans le même esprit, Gustave était déjà allé en 1824 à Berlin, où il avait rencontré Hegel, à qui il avait également remis un exemplaire du Plan de 1822
} 
Guizot, Tocqueville. Grâce à eux, Mill était tenu remarquablement informé de ce qui se passait en France, ce qui n'était pas le cas de la plupart de ses compatriotes et faisait de lui un des meilleurs spécialistes des affaires françaises. Une comparaison entre les lettres à d'Eichthal et les lettres à l'auteur de la Démocratie en Amérique éclaire le caractère tout spécial qui le liait au premier. La correspondance avec Tocqueville montre une estime réciproque, des convictions et des intérêts communs, mais non une véritable amitié : les deux hommes gardaient toujours une certaine réserve et ne se livraient pas.

Dans le second cas, les lettres écrites entre 1829 et 1832, les plus longues (elles occupent plus de la moitié du volume), nous apportent un témoignage exceptionnel sur un moment crucial pour les deux correspondants. Mill venait de rompre avec les benthamites et était donc en quête de nouveaux horizons; mais c'est aussi l'apogée du mouvement saintsimonien, l'époque des prédications de la rue Monsigny, du Globe, puis de la retraite à Ménilmontant. Gustave, qui vient d'adhérer au mouvement, n'aspire qu'à faire partager ses nouvelles convictions et tente donc de «convertir» Mill. Bien plus, de décembre 1831 à Mars 1832, en compagnie de Duveyrier, il entreprend un nouveau voyage en Angleterre, dans le seul but cette fois de recruter de nouveaux adeptes. Dans sa lettre du 30 novembre 1831, Mill se montre extrêmement réceptif aux idées mises en avant par le jeune prosélyte. S'il refuse d'adhérer et prend soin de bien marquer ses distances, il fait tout son possible pour aider d'Eichthal et lui indique ce qu'il convient de faire, de ne pas faire, qui contacter...

La période où, à ses propres dires, Mill tenait chez lui «bureau de saint-simonisme » (Lettre du 1er mars 31, p. 117) n'eut qu'un temps. En 1835, après la publication de La démocratie en Amérique, il entra en contact avec Tocqueville et les affinités politiques entre les deux hommes sont à l'évidence beaucoup plus grandes. L'influence du saint-simonisme sur l'auteur du Système de logique n'en fut pas moins profonde, ainsi que l'atteste l'Autobiographie. Le fait illustre une des propriétés les plus remarquables de la pensée de Mill : sa plasticité, la facilité avec laquelle il pouvait s'assimiler les idées qui lui paraissaient fécondes, tout en gardant entière sa liberté de jugement. Ce n'est pas le moindre mérite de ces lettres que de le voir s'expliquer sur ce point et formuler une règle de conduite intellectuelle, relative au "danger des vues partielles », à laquelle il se conforme le plus scrupuleusement possible. Non content de l'appliquer aux vues des saint-simoniens, elle dicte aussi son jugement sur la société anglaise de son temps. Mill avait hérité de Bentham une profonde hostilité à l'égard de l'Establishment. C'est ainsi que, tout en reprochant à Comte son esprit de système, qui le conduit à ignorer la spécificité de l'Angleterre, il ne ménage pas ses critiques vis à vis de ses compatriotes et dénonce les maux qui accompagnent les progrès de l'industrialisation.

Cette attention scrupuleuse aux faits dans leur complexité explique que l'influence de la pensée politique de Mill se soit exercée dans plusieurs directions. Son enracinement dans la grande tradition libérale ne l'a pas empêché d'être aussi un des pères du socialisme anglais. Les sympathies saints simoniennes de Mill suffisaient pour rendre son libéralisme suspect pour quelqu'un comme Hayek, qui lui préférait celui de Lord Acton.

\section{?./ ?/ ?/}

La correspondance a été publiée par son fils Eugène en 1898 :

John Stuart Mill : Correspondance inédite avec Gustave d'Eichthal (1828-1842) (18641871)/ avant propos et traduction par Eugène d'Eichthal ; Paris, Alcan, 1898.

Elle figure également dans les Collected Works of John Stuart Mill, 33 vols., sous la direction de John Robson; Toronto, Toronto UP, 1963-1991), aux volumes 12, 13, 15, 16 et 17. 
Nous reproduisons le texte de l'édition de 1898, où les lettres qui, à l'époque saintsimonienne, étaient écrites en anglais, sont traduites en français.

\section{CORRESPONDANCE}

IIe Lettre

15 mai 1829

Mon cher d'Eichthal,

[...] Je suis heureux d'apprendre que vous avez tant avancé votre ouvrage sur l'Angleterre. Vous avez très naturellement été frappé de la supériorité des Anglais sur les Français en tout ce qui touche aux qualités qui permettent à une nation de tirer le meilleur parti possible de ses ressources productives et commerciales. Mais cette supériorité se rattache de très près au pire défaut de notre caractère national, à savoir la tendance à tout sacrifier à l'accumulation des richesses et cet égoïsme exclusif et âpre qui l'accompagne. Ceci, je le sais bien, est dû en grande partie à nos institutions politiques qui donnent tout à la fortune et presque rien à la pauvreté. Mais je crains que l'esprit commercial, parmi toutes ses heureuses conséquences, n'entraîne aussi, partout où il prévaut, une certaine dose de ce mal, car ce qui occupe nécessairement le temps, les pensées d'un homme pendant la plus grande partie de sa vie prend sur son esprit un ascendant hors de proportion avec son importance réelle. Là où la poursuite de la fortune, en dépassant ce qui est nécessaire au confort de l'existence, où cette poursuite exclusive, par un individu, de ses intérêts et de ceux de sa famille, devient l'objet essentiel de sa vie, presque toujours cet individu devient incapable d'étendre ses sympathies et le cercle des objets auxquels il s'attache, au delà de sa famille et de lui-même. Vous avez dû observer durant votre séjour ici, bien que nous n'ayez fréquenté que la meilleure classe de la nation, combien il est difficile d'intéresser les gens à quoi que ce soit, qui n'ait pas quelque rapport avec ce qu'ils nomment leur avancement dans la fortune. On trouve dans tous les pays des hommes, arrivés à un certain age, devenus égoïstes et positifs ; mais il y a peu de pays où, comme dans celui-ci, même parmi les jeunes gens, le plus grand nombre soit ouvertement ainsi. En France et en Allemagne, les entraînements ridicules de sentiment et d'enthousiasme sont communs, et rares les excès de froideur et d'égoïsme. Ici c'est le contraire ; il faut beaucoup de tact et de flair social pour se montrer profondément et sérieusement attaché à un objet désintéressé quelconque, sans se rendre ridicule. L'étiquette de ce qu'on appelle la bonne société est de paraître parfaitement insensible à toute impression extérieure ou intérieure. Vous dites que vous n'osez pas penser à la grandeur de notre nation une fois débarrassée de notre bigoterie. Je ne crois pas, pour ma part, que la bigoterie ait été ou soit le pire de nos défauts. C'est de l'indifférence, de l'insensibilité morale que nous avons besoin d'être délivrés. Je voudrais apercevoir chez nous une chance de progrès dans ce sens, sans révolution politique ou sans un changement dans notre éducation nationale, tel qu'une révolution serait, je le crains, nécessaire pour le produire. Vous êtes, en France très en avance sur nous : il vous suffit d'enseigner aux hommes ce qui est bien pour qu'ils le fassent. Ils sont sans instruction, mais sans préjugées, et ils sont curieux et ardents à apprendre. Ici, la grande difficulté est de donner le désir d'apprendre. On a une telle opinion de sa propre sagesse qu'on n'a pas l'idée qu'on puisse s'instruire. On considère trop peu les autres peuples pour beaucoup s'inquiéter si on est au courant ou non de ce qui intéresse seulement la nation ou l'humanité en général. En outre, notre classe moyenne n'a qu'un objet dans la vie: singer ses supérieurs pour lesquels elle a une 
admiration béate et sotte et qui s'attache plus aux défauts qu'aux qualités, celles-ci étant plus difficiles à comprendre et à imiter. Ceux qui désirent bien faire peuvent, il est vrai, grâce à l'esprit d'association que vous admirez tant, obtenir pus, proportionnellement, à leur nombre, qu'en France. C'est un grand défaut de vos compatriotes de s'entourer, comme vous le dites, d'une atmosphère de vanité personnelle qui leu fait désirer de garder tout l'honneur de chaque action pour eux seuls, et les éloigne de recourir à la coopération des autres par crainte d'être obligés d'en partager le succès avec eux.

Le défaut de vos hommes publics est certainement le désir d'éblouir. Ici, on s'en occupe peu. Les bravos « qui ne mènent à rien » sont peu appréciés. Mais le succès qui a une valeur échangeable, qui se traduit en livres sterling, ou en puissance tangible, celui qui vient des éloges des clases gouvernantes, est hautement estimé et recherche aussi ardemment ici que partout ailleurs. Nous faisons sans doute de rapides progrès, principalement, il est vrai, en en nous dépouillant de nos préjugés. Nous ne considérons plus nos institutions comme le meilleures, mais nous commettons de lourdes erreurs chaque fois que nous essayons de les corriger.

C'est cependant quelque chose d'avoir le champ libre. Les idées saines et le bon sens appliqués aux affaires publiques ont, je crois, plus de chances d'être écoutés et d'exercer leur influence en ce moment qu'à aucune autre époque depuis cent ans. Mais où les rencontrer?

Je vais seulement me mettre à lire le livre de M. Comte que vous m'aviez recommandé. Je serais très désireux, quand je l'aurai lu, de discuter avec vous les principes du parti du «Producteur », surtout en ce qui concerne «l'hérédité de la propriété »: la doctrine de ce groupe, sur ce point, telle que vous l'exprimez, me paraît une grande hérésie : mais je ne puis naturellement dire ce que j'en pense avant de savoir de quelles réserves et de quelles restrictions ils l'accompagnent. Je pense, avec M. Bentham, que la propriété devrait échoir à l'Etat plutôt qu'aux héritiers collatéraux quand il n'y a pas de testament. Vous avez probablement vu d'après nos débats que la proposition d'introduire une taxe pour les pauvres en Irlande est sérieusement agitée par le gouvernement. Le progrès de l'opinion sur ce sujet, depuis quelques années, est très saisissant. Je crois que la discussion sur ce point sera extrêmement instructive, et bien digne de l'attention de nos hommes d'Etat.

Croyez moi votre sincèrement dévoué,

J. St. MILL

\section{IIIe Lettre}

Londres, 8 octobre 1829

Mon cher d'Eichthal,

J'ose à peine espérer que vous me pardonnerez mon long silence, surtout après votre désir si chaleureusement exprimé non seulement que nous nous écrivions, mais encore que nous discutions entre nous certains sujets sur lesquels il importe que vous, moi et tous les hommes aient des idées justes. De fait, le loisir m'a longtemps manqué pour lire les livres que vous avez eu l'amabilité de me laisser, et depuis j'ai eu trop d'autres occupations pour pouvoir vous écrire. Il m'a été impossible jusqu'ici de lire aucun article du Producteur : je ne puis par conséquent vous donner une opinion sur les Doctrines de l'Ecole saint-simonienne, aussi complète que je pourrai peut-être par la suite. Cependant autant que j'en puis juger jusqu'ici, si jamais je devais devenir prosélyte de cette école, ce serait plutôt d'après les principes de votre dernière lettre, que d'après aucun des livres que j'ai lus. Dans votre lettre il n'y a que peu de choses, bien qu'il y en ait, sur lesquelles je sois en désaccord avec vous. Je ne puis en dire autant quant aux livres; lorsque j'ai lu les «Opinions littéraires, 
philosophiques et industrielles ${ }^{6}{ }^{6}$, desquelles je me promettais un grand profit, j'ai été profondément surpris de trouver une œuvre aussi superficielle. Cela m'a paru être le fait d'hommes qui n'avaient ni lu ni pensé, mais qui avaient jeté à la hâte sur le papier les premières notions indigestes qui viendraient à l'esprit d'un écolier frais émoulu. Cependant, lorsque j'ai lu le «Traité de Politique Positive» de Comte $^{7}$, je n'ai plus été surpris de la haute opinion que je vous ai entendu exprimer sur le livre et l'auteur, et j'ai même été amené par la plausibilité de sa méthode, à me former des doctrines qu'il professe une opinion plus haute qu'elles ne me paraisse, après réflexion, le mériter. Je trouve à sa philosophie les mêmes défauts que ceux qu'il reproche à la philosophie du XVIIIe siècle. La partie critique seule m'en paraît solide. La partie organique me semble soulever cent objections. Elle abonde en observations très ingénieuses, mais telles que le progrès des événements en suggère en ce moment à tous les esprits au niveau du siècle, en Europe ; mais c'est une grande erreur et très commune aussi dans laquelle cette secte semble en grand danger de tomber, de supposer que quelques observations frappantes et originales suffisent à fonder une science positive. M. Comte est un auteur extrêmement clair et méthodique, de style très agréable, et il enchaîne si bien ses idées que parfois la cohésion parfaite et la consistance logique de son système donnent l'illusion de la vérité. Cette faculté de systématiser, de poursuivre un principe jusqu'à ses dernières conséquences, et celle d'exposer d'une façon claire et suivie, qui l'accompagne généralement, me paraissent être les qualités caractéristiques de tous les bons écrivains français : elles se rattachent étroitement à leur défaut caractéristique qui me semble être celui-ci : ils sont tellement satisfaits de la clarté avec laquelle leurs conclusions découlent de leurs prémisses, qu'ils ne s'arrêtent pas à comparer les conclusions mêmes avec les faits : et cependant c'est seulement lorsqu'elles ont subi l'épreuve de cette comparaison, que nous pouvons être certains qu ces prémisses contenaient tout ce qui est essentiel à la question. Ils déduisent la politique, comme les mathématiques, d'une série d'axiomes et de définitions, oubliant qu'en mathématiques le danger de vues partielles n'existe pas : là une proposition est vraie ou ne l'est pas; et si elle est vraie, nous pouvons en toute sûreté l'appliquer à tous les cas qu'elle comprend dans ses termes. Mais il est loin d'en être ainsi de la politique et des sciences sociales ; ici, l'erreur provient rarement de ce que nous posons des prémisses fausse, mais généralement de ce que nous négligeons d'autres vérités qui liment et modifient la portée des premières. Aussi il me semble qu'on peut imputer à beaucoup de philosophes français le défaut que Cousin reproche à Condillac, de n'insister que sur un seul côté de la question, alors qu'il y en a plusieurs, ou de n'apercevoir un objet que par une de ses faces, d'un unique point de vue, alors qu'il en est plusieurs autres également essentiels à la juste appréciation de cet objet. Si je voulais relever tous les cas où ce défaut existe dans l'oeuvre de Comte, je pourrais le faire à chaque page, car il envahit tout le livre et c'est, je crois, ce défaut qui permet à Comte de donner à ses idées cette forme liée et systématique, par laquelle elles prennent l'apparence d'une sorte de science positive.

Et d'abord je prends le principe fondamental de tout le système : que le gouvernement et l'union sociale existent pour concentrer et diriger toutes les forces de la société vers une seule fin.

L'auteur ne peut vouloir dire que le gouvernement ne doit pas exister pour plus qu'un objet unique, ni que cet objet unique doit être de diriger les forces unies de la société vers une seule fin. Quel fondement pour un système de science politique! Le gouvernement existe en vue de réaliser tout ce qui concerne le bien de l'humanité, et le plus élevé et le plus important des objets qu'il poursuit c'est le progrès de l'homme lui-meme comme être moral

\footnotetext{
${ }^{6}$ Publication saint-simonienne de 1824 ; reproduite dans : Saint-Simon, Oeuvres complètes, Paris, PUF, 2012 , T. IV, p. 3027-3144.

${ }^{7}$ Voir n. 2.
} 
et intelligent, ce qui n'est pas compris dans les catégories de M. Comte. Les forces unies de la société n'ont jamais été et ne pourront jamais être dirigées vers un seul but, et je ne vois pas de raison de désirer qu'elles le soient. Les hommes ne viennent pas au monde pour accomplir une fin unique, et il n'existe pas une fin unique qui, réalisée de la façon la plus complète, puisse les rendre heureux. Quand M. Comte recherche quel est ce but unique, il trouve soit la domination de l'homme sur l'homme, qui est conquête, ou la domination de l'homme sur la nature, qui est la production : la première a été le but de la société dans l'antiquité et les temps féodaux (ce qui n'est nullement vrai!). Mais ce passé n'existe plus, et par suite c'est la production qui doit être le but de la société actuelle.... Et pourquoi, je vous prie ? La conquête et la production sont-elles les seuls objets qu'on puisse concevoir comme devant être poursuivis par les efforts combinés des être humains ? Voici une des mille raisons qui prouvent que la philosophie de Saint-Simon ne pouvait prendre naissance qu'en France.

Si M. Comte était né en Angleterre, où depuis un siècle cette idole de «la production » a été placée sur l'autel et adoré avec dévotion, s'il avait vu combien l'importance disproportionnée qu'on lui attribue est la vraie racine de nos pires vices nationaux, corrompt les actes de nos hommes d'état, les doctrines de nos philosophes, et endurcit l'âme de notre peuple au point qu'il faut renoncer à lui insuffler aucune ardeur d'intelligence ou de cœur, il aurait compris qu'une philosophie qui fait de la production l'unique but de l'union sociale, rendrait irrémédiables les grands maux sociaux qui menacent partiellement l'état actuel de la civilisation. Par là, on arrive aussi à la grande conclusion pratique de l'école saintsimonienne, que la direction du gouvernement doit être mises aux mains des principaux industriels, le pouvoir temporel du moins, le pouvoir spirituel devant revenir aux savants et artistes. Je ne sais pas comment cela serait en France; mais je sais qu'en Angleterre ce sont là les classes de personnes qui se distingueraient par l'esprit le plus borné et bigot, par les vues les plus étroites et les plus basses, touchant tout ce qui dépasserait leurs affaires ou leurs familles. J'ai encore mille choses à dire à ce sujet, mais je dois passer à une autre question.

Il n'y a, suivant M. comte, qu'une seule loi du développement de la civilisation humaine. Vous, qui avez été en Angleterre, vous pouvez dire si c'est vrai. N'est-il pas manifeste que ce ces deux nations, l'Angleterre et la France, sont des exemples du progrès de la civilisation réalisé par deux voies différentes, sans que l'une d'elles ait jamais passé ou doive sans doute jamais passer par l'état que l'autre a traversé ? Seuls les animaux inférieurs n'ont qu'une loi, celle de leur instinct. L'ordre du développement des facultés de l'homme est aussi variable que les conditions où il est placé. Il est triste de constater chez un homme comme M. comte, combien toutes ses vues sur l'histoire ont été faussées et déformées par la nécessité de prouver que la civilisation n'obéit qu'à une loi, la loi du développement progressif; combien il en a été aveuglé sur les mérites des Grecs et des Romains et le défauts $d u$ moyen âge : parce qu'il y a eu des progrès sur certains points, à telles époques, il croit qu'il y a eu progrès sur tous les autres.Pourquoi ne pas admettre que, si l'humanité a progressé sur certains point, elle a rétrogradé sur d'autres?

Il n'y a positivement pas de place pour l'Angleterre dans le système de M. Comte ; il a énuméré tous les modes d'état social qui peuvent exister et il n'y en a pas un seul dans cette énumération qui convienne pour l'Angleterre.

Malgré toutes ces objections, le livre de M. Comte abonde en observations excellentes et justes; et si l'on voulait se contenter d'en prendre une partie et de laisser le reste, ces doctrines, en y pratiquant les corrections et modification nécessaires, auraient grande valeur. Mais si les prosélytes de Saint-Simon persistent à former une secte, ce que des penseurs indépendants devraient éviter par dessus tout, et s'ils croient devoir, en tant que secte, en accepter tous les dogmes sans distinction et sans restriction, non seulement ils ne feront aucun bien mais ils feront, je le crains, un mal immense : de substituer un fragment de la 
vérité à un autre, ce n'est pas là ce qui est désirable, mais bien de grouper ces fragments de façon à en obtenir la plus grande somme possible de vérité.

Je ne me suis pas réservé assez de place pour vous dire le plaisir que j'ai eu à lire votre brochure qui m'a presque complètement converti, et les quelques pages de votre livre sur l'Angleterre que votre frère m'a montrées. Toutefois je vous écrirai par l'entremise de votre ami, j'espère pouvoir dire maintenant notre ami, M. Victor Lanjuinais ${ }^{8}$, que je souhaite connaître davantage à son retour d'Ecosse ; ce que j'ai vu de lui me plaît extrêmement.

En attendant croyez moi

votre sincèrement dévoué,

J.St. MILL.

\section{IVe LETTRE}

India House, Londres, 7 novembre 1829

Mon cher d'Eichthal,

[Mill entretient son correspondant d'un projet de périodique, qui n'aboutira pas, mais pour lequel il demande sollicite la collaboration de son correspondant]

[...] Je ne me suis pas réservé assez de place pour vous parler longuement de l'école saintsimonienne, et, de fait, ayant été absent, je n'ai pas encore eu l'occasion de lire un seul article du Producteur. Je désire principalement, surtout pour le moment, vous parler des choses que j'approuve et j'admire dans cette école, car je crains que ma dernière lettre ne vous ait laissé l'impression que mon opinion sur ce sujet fut moins favorable qu'elle ne l'est en réalité actuellement. Donc, en premier lieu, j'approuve et je loue hautement un des points des plus importants articles du système, dont l'école a fait un article de foi, la nécessité d'un pouvoir spirituel. Elle a été érigée comme une fin dernière vers laquelle nous tendons, et que nous atteindrons un jour, un état dans lequel la masse du peuple, c'est-à-dire le illettrés, aura pour l'autorité des savants, en morale et en politique, les mêmes sentiments de déférence et de soumission qu'elle a actuellement pour les doctes en sciences physiques. C'est là, j'en suis persuadé, l'état d'équilibre de l'esprit humain, et l'idée fixe que nous devons le considérer comme notre fin suprême, contribuera beaucoup à nous préserver de nombreuses erreurs dans lesquelles sont tombés les philosophes du XVIIIe siècle, et auxquelles sont exposés tous ceux qui croient que la diffusion de la science parmi les classes ouvrières et le progrès des intelligences qui en résulte, doit être le grand instrument de la régénération de l'espèce humaine. Cependant, avant que l'on puisse atteindre cet état ou même y viser, il sera nécessaire de réaliser plusieurs progrès importants dans l'organisation sociale; et tout d'abord la suppression des grands intérêts sociaux égoïstes. En effet, tant qu'ils subsistent, ceux qui, sans eux, formeraient la classe instruite, n'ont pas de raison de chercher à acquérir une réelle instruction politique et morale, et sont victime de préjugés dont sont exempts ceux qui s'adonnent aux sciences physiques. Ils peuvent réussir dans une industrie en ignorant les autres ou en nuisant aux autres; ils peuvent écrire en faveur des classes qui ont des intérêts égoïstes, et servent leur égoïsme et leur aigreur ; ou, s'ils s'adressent au menu peuple, ils peuvent trouver dans le mécontentement fondé de la foule contre les institutions de quoi se faire une popularité sans éclairer les intelligences ou entretenir les esprits de bonnes habitudes de sentir ou de juger. D'autre part, je m'élève contre le moyens que les saint-simoniens proposent pour organiser le pouvoir spirituel. Il me semble que vous ne pouvez pas du tout l'organiser ; qu'est ce que le pouvoir spirituel, sinon l'influence de l'esprit sur l'esprit? Les instruments en sont la communication

\footnotetext{
${ }^{8}$ Fils du célèbre constituant, lui-même depuis député et ministre.
} 
individuelle, la chaire et la presse. Si vous tentez de grouper les savants, il vous faudra quelqu'un pour les choisir et établir leurs titres; en quoi cela différera-t-il alors d'une assemblée nationale élective avec une condition d'éligibilité, une condition non de propriété, mais d'instruction? Le second grand service que les saint-simoniens ont à mon avis rendu est celui-ci : s'ils n'ont pas été les premiers à observer, car tout le monde y a assurément plus ou moins pensé, ils ont du moins démontré plus abondamment que personne et mis plus en relief que ne l'avait fait jusqu'ici aucun philosophe, ce fait que les institutions qui, considérées en elles-mêmes, sont, sans contestation possible, impropres à produire autre chose que les plus effroyables maux (l'église Catholique par exemple) ont pu cependant, à un stade particulier du progrès de l'esprit humain, être non seulement hautement utiles, mais absolument indispensables, constituer la seule voie par laquelle l'esprit humain ait pu progresser et atteindre un degré plus élevé de son développement. Tenir un juste compte de cette grande vérité est le résultat de vues étendues sur l'histoire de l'humanité, mais aussi une condition nécessaire pour obtenir ces vues. Sans elle, il n'est pas possible de considérer et de juger les temps passés avec impartialité ou de les soumettre à une autre mesure que celle du temps présent ; or, c'est s'exposer à juger le présent aussi mal que le passé, car il y a assurément à chaque époque présente beaucoup de choses bonnes pour cette époque, bien que mauvaises pour l'être humain vivant à une autre époque, peut-être même à toute époque autre que celle où elle s'applique ; et quiconque ne fait pas cette distinction est un mauvais philosophie pratique même pour son propre temps : car celui qui ne fait pas de distinction dans le passé ne peut pas en faire dans le présent.

A chaque époque, il y a des pays et dans chaque pays des hommes à tous les degrés possibles de la civilisation, depuis les plus bas jusqu'aux plus élevés que l'humanité ait atteints à cette époque ou dans ce pays; et cependant l'on trouve difficilement un seul homme qui ne pense ou parle habituellement comme si ce qui est bon ou mauvais pour une partie du pays ou des individus, devait être bon ou mauvais pour toutes les autres parties. Il est peu probable qu'une personne imbue de l'esprit des saint-simoniens tombe dans cette erreur. Ils ont, et leur système me paraît même tendre à produire à l'excès, cet éclectisme et cette libéralité de compréhension qui, par là même qu'ils élargissent à la fois nos idées et nos sentiments, sont beaucoup plus excusables et bien moins nuisibles, même exagérés, que le défaut inverse. Ils ont la disposition la plus contraire à l'esprit de critique et de discussion, celle qui nous engage à ne pas combattre, mais à passer outre sur les erreurs de ce qu'on nous présente, en vue de saisir et de nous approprier cette portion ou cette parcelle (bien que minime) de la vérité, qui doit nécessairement se trouver à l'origine de toute erreur, si celle-ci n'est pas une simple faute de raisonnement. Le grand danger pour l'humanité n'est pas de voir ce qui n'est pas, mais de négliger ce qui est ; des hommes capables et intelligents se trompent rarement par suite de la première de ces causes ; mais leur puissance d'esprit ne les protège pas contre les maux provenant d'une vue imparfaite et partielle de la réalité ; ce sont les demi-vérités et non les erreurs qui sont le fléau du progrès humain ; et de là il résulte que le vrai mode de philosophie et de discussion pour un homme qui poursuit le bien de l'humanité et non la satisfaction de sa propre vanité, doit être l'opposé direct de la philosophie critique du siècle dernier. Il doit consister non à combattre les fausses opinions des hommes, mais à acquérir cette science qui nous permettra de s'en former de justes, lesquelles chasseront les fausses, comme les nouvelles feuilles du printemps chassent les feuilles sèches de l'automne.

Le grand instrument du progrès des hommes est de leur fournir l'autre moitié de la vérité dont ils n'ont connu qu'une face; de leur présenter le côté blanc de l'écusson qu'ils prétendent voir parce qu'il n'en ont vu que le côté noir. Les véritables croyants, même en fait de saines croyances, trouvent insuffisante la faible action qui, souvent même, est une action nulle, qu'on a sur un homme, en lui inculquant simplement une opinion. 
Une opinion suggère difficilement quelque chose à l'esprit ignorant ; elle peut devenir un mot d'ordre, mais elle ne sera jamais un principe d'action vibrant et vivant en lui. Les mots, ou ce qui peut être exprimé par des mots, ne profitent qu'aux esprits auxquels les mots suggèrent une série d'idées correctes et claires, et chez qui ils réveillent une connaissance saine et exacte préalablement acquise des choses désignées par les mots. Aussi est-il peu utile de modifier les opinions des hommes, et même il est souvent très dangereux de les troubler, jusqu'à ce qu'on ait amené leur esprit à ce haut état de culture, qui produit naturellement et presque spontanément des opinions plus saines.

D'ailleurs, pour obtenir ce résultat, nous ne devons pas attaquer en masse leurs opinions présentes, mais rechercher avec attention ce qu'elles contiennent de bon et tenter de les diriger de là et par là vers quelque chose de mieux. C'est là de l'éclectisme pratique.Sans le vouloir, je viens de développer longuement une des parties les plus intéressantes de la philosophie saint-simonienne, quoiqu'elle ne lui appartienne pas exclusivement, à savoir, la distinction entre la partie critique et la partie organique de toute philosophie, et entre les époques critiques et organiques de l'esprit humain. Les saint-simoniens ont eu aussi, je pense, le grand mérite d'avoir signalé comme le premier pas à faire dans la recherche des vérités de la politique pratique, l'effort qui consiste à reconnaître le caractère de la prochaine étape par laquelle, dans l'ordre naturel du progrès des civilisations, la nation en question doit passer ; ce premier pas nous permet ensuite de donner à nos efforts comme but suprême la tâche de faciliter la transition de la société vers son nouvel état.

Ceci posé, il s'ensuit que nous devrons souvent maintenir ou même établir des institutions propres à produire de grands maux, lesquels maux dans d'autres états sociaux seraient peutêtre sans mélange, à condition que ces institutions soient en même temps de nature à corriger d'autres tendances nuisibles, qui pourraient l'emporter ou être plus redoutables à cette même époque, autrement dit, dans ce même état de l'esprit humain. En ces matières, je diffère des saint-simoniens, principalement sur un point : ils paraissent croire que la plupart des esprits, par une sorte de fatalité ou de nécessité, accroissent ou développent leurs diverses facultés toujours dans un ordre déterminé, comme le corps humain, et de là ils concluent que nous devons toujours soit être immobiles, soit avancer, soit reculer. Pour ma part je suis convaincu qu'un examen plus approfondi de la question montrerait que des nations différentes, et aussi bien des esprits différents, peuvent progresser et en réalité progressent par différentes voies; que les nations et les hommes, bien qu'à peu près au même degré de civilisation, peuvent cependant être de caractères très variés, et que des changements peuvent se produire chez un homme ou chez une nation, qui ne sont ni des pas en avant ni des pas en arrière, mais une marche oblique. Je vous ai dit, je crois, tout ce que j'ai à dire pour le moment sur l'école saint-simonienne. [...]

Très sincèrement vôtre, J. St. MILL

\section{VIIIe LETTRE}

Londres, 30 novembre 1831

Mon cher d'Eichthal,

Je vous connais trop bien pour vous parler d'autre chose que de l'oeuvre vraiment grande et apostolique à laquelle vous vous êtes voué, et que pour ma part, bien que je sois loin être entièrement d'accord avec vous, je me suis habitué à considérer comme l'œuvre de régénération sociale sans contredit la plus importante qui se poursuive aujourd'hui. 
Je vous suis très reconnaissant à vous et à vos associés d'avoir bien voulu me croire digne de recevoir le Globe ${ }^{9}$. Quand bien même je n'aurais envers vous d'autre sympathie, ce serait toujours pour moi un noble spectacle que celui d'une troupe de jeunes hommes marchant, comme vous faites, le front levé et défiant le monde.

Ma lecture journalière du Globe jointe à diverses autres causes m'a fait sensiblement me rapprocher de beaucoup de vos opinions, et je vous regarde comme étant décidément à la tête du mouvement de la civilisation.

Je suis maintenant disposé à croire que l'organisation sociale que vous avez conçue, sauf les modifications que l'expérience pourra vous suggérer, deviendra probablement l'état final et permanent de l'espèce humaine. Seulement, je diffère de vous en ceci qu'il faudra, je crois, bien des siècles pour amener l'humanité à ce point. En attendant, il faudra se contenter d'approximations, au moyen de ces changements graduels, de ces moyens transitoires qui sont si admirablement indiqués et discutés dans vos écrits, et qui, indépendamment de ce qu'ils préparent, sont d'incontestables et positives améliorations. Votre système, fallût-il même le considérer comme impraticable, différerait cependant de tous les autres systèmes auxquels on a pu faire le même reproche, sous ce rapport que, si le but final ne peut être atteint, ici du moins tous ou presque tous les avantages pratiques se trouvent sur le chemin qu'on suit.

Vous pensez, je le sais, que tous ceux qui adoptent votre système peuvent par là même qu'ils sont capables de suffire à tout ce qu'il devrait exiger d'eux, s'il devenait universel. Ce n'est pas mon avis ; mais puisque c'est le vôtre, c'était mon devoir de commencer, comme vous l'avez fait, l'expérience de réalisation sur l'échelle où il vous était permis de le faire. Je suis cette expérience et la suis avec toute la sollicitude et l'anxiété d'une personne qui y rattache toutes ses espérances d'une prompte et rapide amélioration de la société humaine.

Si des hommes dont les vues sont si larges, l'enthousiasme si ardent et si généreux, l'intelligence si pénétrante et si vigoureuse, devaient être incapables de réaliser leur propre conception du devoir, quelle espérance resterait-il pour le reste de l'humanité ? Si au contraire la société saint-simonienne se maintient sans schisme et sans hérésie, si elle continue à propager sa foi, et à multiplier le nombre de ses sectateurs, dans la proportion de ces deux dernières années, et cela pendant quelques années encore, alors je verrai comme un rayon de lumière luire à travers les ténèbres. Mais alors même qu'il n'en serait pas ainsi, ce qui s'est fait ne serait pas perdu. Je ne désespérerai pas plus que vous ne devrez le faire, mais ce sera une douloureuse chute de nos espérances.

Ecrivez-moi quelquefois, mon cher ami, ne craignez pas que votre peine soit perdue. Je n'ai jamais lu un seul article du Globe qui n'ait eu son effet sur moi, et par lequel je ne me sois plus ou moins senti amélioré.

Et si l'heure était venue pour l'Angleterre (si aujourd'hui il n'était pas aussi impossible de faire accepter parmi nous aucune vue organique, que cela aurait été le cas pour votre maître Saint-Simon dans le paroxysme de la Révolution française), si le moment, dis-je, était venu, je ne sais si je ne renoncerais pas à toute chose au monde pour devenir, je ne dis pas un d'entre vous, mais comme vous. Mais notre 10 août, notre 20 juin ${ }^{10}$, peut-être notre 18 Brumaire sont encore à venir. Et qui de nous sera encore debout quand l'ouragan aura passé ?

A vous de cœur,

J. St. MILL.

\section{XIIe LETTRE}

\footnotetext{
${ }^{9}$ Fondé sous la Restauration, Le Globe fut l'organe du saint-simonisme du 11 novembre 1830 au 20 avril 1832.

${ }^{10}$ Le Serment du jeu de Paume a eu lieu le 20 juin 1789.
} 
[L'expérience saint-simonienne s'est terminée, ou plutôt s'est déplacée : après l'échec de Ménilmontant, certains se tournent vers la méditerranée (Egypte, Algérie, Grèce), pendant que d'autres entrent dans la banque et les chemins de fer. Mill, de son côté, a pris ses distances par rapport au mouvement.]

Mon cher Gustave,

India House, 14 septembre 1839

Je suis heureux de vous lire de nouveau, après une si longue interruption de notre correspondance. J'ai reçu votre dernière brochure ${ }^{11}$ et l'ai lue avec l'intérêt que j'apporte à tout ce qui vient de vous. J'y trouve, comme dans Les Deux Mondes, des preuves de ce qui me paraît une importante vérité. Je suis convaincu depuis longtemps que non seulement l'Orient comparé à l'Occident, mais la race noire comparée à la blanche, s'en distinguent par des traits qui se rapprochent de ceux que vous définissez; que ce n'est pas les premiers seulement, mais nous qui aurions à profiter grandement d'une familiarité plus intense et plus sympathique ; que si notre intelligence est plus développée et notre activité plus intense, les noirs possèdent exactement ce qui nous est indispensable comme contrepoids, dans leur amour du repos, et dans leur aptitude supérieure pour le plaisir animal, source d'une grande sensibilité de sympathie, qui est caractéristique chez la race nègre.

Je pense depuis longtemps aussi que la même distinction existe, bien que d'une façon moins accentuée, entre les peuples du Nord et du Midi de l'Europe : le Nord est destiné à être l'atelier matériel et intellectuel de l'Europe, le Midi son «palais de plaisir »; ni l'une ni l'autre des parties ne remplira sa fonction utile à l'une et à l'autre, tant que nous ne travaillerons pas pour les peuples du Midi, et que nous, dans la mesure de notre tempérament, nous ne nous approprierons pas leur sens du plaisir sensuel et pompeux.

Je suis, vous le voyez, très bien préparé à accueillir favorablement vos méditations et à me joindre à vos aspirations : je suis aussi désireux que jamais de conserver avec vous cette sorte de communion intellectuelle dont j'ai joui depuis longtemps. Je ne sens nullement s'éteindre la jouissance que j'éprouve à la spéculation philosophique, bien que j'aperçoive de moins en moins la possibilité d'amener un certain nombre de personnes à s'associer sous le drapeau et au nom d'une série de principes déterminés. Mais la bonne semence n'est jamais perdue : elle prend racine, et surgira quelque part : elle servira à la reconstruction des idées du monde civilisé auquel le nôtre n'est qu'une période de préparation, mais à la naissance duquel toutes les choses et tous les hommes du présent travaillent, bien que les hommes en soient en général aussi inconscients que les choses : «C'est pourquoi, jetez votre pain sur les eaux, et vous le retrouverez après bien des choses ».

J'ai été très peiné d'apprendre la maladie de votre père et l'indisposition d'Adolphe. Assurez-les tous les deux de mes souvenirs affectueux, et dites-moi, quand vous m'écrirez, très exactement comment ils seront.

Votre très affectionné, J.St. MiLL.

\section{XXVe LETTRE}

Mon cher d'Eichthal,

Saint Véran, Avignon; le 20 août 1866

11 Lettres sur la race blanche et la race noire, Paris, Paulin, 1839. Deux ans plus tôt, d'Eichthal avait exposé ses idées sur l'avenir de l'Orient dans Les Deux Mondes, servant d'introduction à un ouvrage de M.

Urquhart :La Turquie et ses ressources, Leipzig, F. A. Brockhaus, 1837. 
Votre lettre n'est parvenue à Londres qu'après mon départ. Depuis bientôt huit jours, je suis ici, et bien content, je vous jure, de pouvoir revenir à des occupations tranquilles. La vie parlementaire fatigue et dissipe l'esprit toujours pour l'exercer quelquefois.

C'eût été un vrai plaisir pour moi que de causer avec vous et de comparer nos impressions. Je suis très indifférent aux pensées de ceux pour qui les événements du moment ne sont que des événements d'un moment ${ }^{12}$; quand même ce moment s'appellerait un siècle. Mais ceux chez qui tout ce qui arrive se lie à une conception générale du développement humain - et c'est notre cas à tous deux - ceux-là ont toujours quelque chose à dire l'un à l'autre. Espérons que l'occasion nous en viendra.-Bien des amitiés à votre frère, et à Duveyrier, dont la santé altérée me fait de la peine.

Votre bien dévoué

J. St. MILL

\section{XXXIVe LETTRE}

Avignon, le 10 mai 1870

Mon cher d'Eichthal

Je suis ici pour quelques semaines, et je serai charmé d'y recevoir l'annuaire de l'Association $^{13}$. Je vous envoie par mandat de poste ma souscription annuelle.

Vous avez été heureux, c'est à dire clairvoyant, dans vos prévisions politiques au commencement de la crise actuelle, et c'est là une forte raison pour ajouter foi à vos prédictions d'aujourd'hui. Cependant j'ai grande envie de savoir sur quoi repose la supposition que l'issue de la situation actuelle sera la république. Viendra-t-elle par un coup de main de la classe ouvrière de Paris et des grandes villes? Alors tout dépend de la fidélité des soldats, qui sont beaucoup plus nombreux et mieux armés et qui seront probablement mieux commandés que dans les révolutions précédentes. Ou bien croyez-vous que les électeurs apprendront à nommer des députés républicains? Cela me semble fort douteux, en ce qui regarde les campagnes; car quoique les paysans désirent, selon toute apparence, un gouvernement libéral, l'élection des maires, etc., je crois que toutes les fois qu'on leur fera croire qu'ils ont à choisir entre Napoléon et la république socialiste, ils voteront pour Napoléon; et il faut avouer que les républicains socialistes ne font rien pour les rassurer.

Votre tout dévoué

J. St. MILL

XXXVe LETTRE

A[vignon], 24 mai 1870

Mon cher d'Eichthal,

[...] Je vous remercie bien des explications que vous m'avez données sur votre prédiction politique. Maintenant que je la comprends mieux, j'en reconnais aisément la justesse. J'avais d'abord crû que vous vous attendiez à un dénouement républicain beaucoup plus prompt. Je crois avec vous que le progrès de l'opinion est dans le sens des convictions républicaines, et cela dans une forme plus élevée et plus profonde que tout ce qu'on entendait par ce mot du temps de notre jeunesse. Vous avez assisté au berceau de ces nouveaux éléments par votre participation au mouvement saint-simonien et ma lettre de $1831^{14}$ montre que dès lors j'ai pleinement reconnu l'importance européenne de ce

\footnotetext{
${ }^{12}$ Allusion à la bataille de Sadowa ( 3 juillet 1866) où la Prusse défit les troupes de l'Empire Austro-Hongrois.

${ }^{13} \mathrm{~L}$ 'association pour l'encouragement des études grecques en France.

${ }^{14}$ Voir supra la lettre du 30 novembre 1831.
} 
mouvement. Mais l'opinion ne peut être assez forte pour prévaloir sur les obstacles que lorsqu'elle sera devenue assez générale pour gagner l'armée. Tant qu'il y aura 7 millions d'ignorants pour voter des plébiscites de confiance et 1 million d'hommes armés prêts à obéir aux ordres de leur chefs, il me semble que nous sommes encore très éloignés du but que sans doute on finira par atteindre.

\section{XXXVIe LETTRE}

Blackheath Park, Kent

le 27 août 1870

Mon cher d'Eichthal

Merci d'avoir pensé à moi dans un temps si douloureux. Depuis longtemps je suis arrivé à la triste conviction que malgré l'incontestable réalité des progrès modernes, nous ne sommes pas encore à l'abri des grands malheurs et des grands crimes que notre siècle se flattait d'être parvenu à bannir de la terre. Je plains profondément le peuple français, qui n'est pas responsable de tout ceci, qui n'aime pas et n'a pas voulu la guerre, et qui est condamné à la payer du meilleur de son sang, et peut-être d'une humiliation nationale la plus difficile à supporter. Pourvu que l'Europe, et surtout la France, apprenne de ces tristes événements, que lorsqu'un peuple abdique la direction de ses propres destinées, et se résigne à ce qu'un gouvernement fasse de lui un simple instrument de sa volonté, il est condamné à supporter toutes les conséquences de ce qu'il a laissé faire en son nom; et qu'un gouvernement qui par les conditions de son existence a besoin de tout ce qu'il y a de plus malhonnête et de plus corrompu dans le pays, finit par être trompé par eux, au point que même son appui de prédilection, l'administration militaire, se trouve pourrie et en décomposition au moment du besoin.

Quelles que puissent être pour la France les suites immédiates de ces événements, il ne lui faudra pas beaucoup d'années pour redevenir tout aussi grande qu'auparavant. Mais elle devra se contenter d'être l'une des grandes puissances de l'Europe, sans prétendre à être la seule, ou même la première: il lui faudra reconnaître pour les relations internationales comme pour celles de la vie civile, le règle de l'égalité. La prétention d'un pays quelconque à être tellement au dessus des autres pour que rien d'important ne se fasse sans le consulter, ne peut plus se soutenir aujourd'hui, et la France devrait voir dans la répudiation universelle d'une telle prétention, le triomphe du principe qui fait sa propre gloire.

J'espère qu'au moins vous n'aurez pas d'autres malheurs que le désastre public à déplorer, et que la guerre épargnera toute votre famille.

Je suis arrivé ici huit ou dix jours avant la déclaration de guerre, alors qu'un pareil coup semblait presque aussi peu probable que la destruction de Paris par un tremblement de terre. La rapidité foudroyante des grands événements d'aujourd'hui n'est pas ce qu'ils ont de moins étonnant.

Votre toujours affectionné

J. St. MILL 